\title{
A MONITORING METHOD OF COLOR DECAYING FOR COLORED PATTERNS IN ARCHITECTURAL HERITAGE
}

\author{
L. Zhang ${ }^{\text {a }}$, J. Xie ${ }^{\text {b }}$, L. Qin ${ }^{\text {c }}$ Z. Liu ${ }^{\text {a }}$, G. Liu ${ }^{\text {a* }}$ \\ a Architecture school of Tianjin University, Tianjin 300072, China - arcdragon@163.com, liuzhe7919@tju.edu.cn, \\ LGLGMIKE@163.com \\ b Tianjin International Engineering Institute, Tianjin University, Tianjin 300072, China -xjltjuhj@tju.edu.cn \\ ${ }^{c}$ Administrative office of the Summer Palace, Beijing 100091, China, - qlei68@126.com
}

KEY WORDS: Heritage Protection, Heritage Monitor, Summer Palace, Colored Pattern, Color Analysis

\begin{abstract}
:
As a special part of architectural heritage, the colored pattern is not only artwork to decorate the architecture, but information reflecting specific historical era. So it has great cultural and artistic value. However, the colored pattern is one of the most sensitive types of cultural relics, which is sensitive to natural environment changes and highly vulnerable to erosion. In order to strengthen the protection of colored patterns in history architectures, it is necessary to monitor the status quo of them.

This work introduces a monitoring method of color decaying for colored patterns in architecture: set up the illuminants, adjust the illuminants, detect the color information and calculate the color difference. Based on the color difference by CIE DE2000 formula, the color decaying status of the pattern can be evaluated. The monitoring period should be at least three months.

About four years' work has been carried out for colored patterns in the Long Corridor of the Summer Palace, and the color differences $\triangle \mathrm{E} 00$ are about from 2 to 5 , some can be over 9 . In result, most colored patterns keep in good health condition. Color decaying happens every time and there are slight changes in most colored patterns. In 2nd quarter 2017, aware changes happened in nearly all the patterns. According to the color difference to evaluate the color decaying of the colored patterns, it is an efficient method to analyze the health status of colored patterns.
\end{abstract}

\section{INTRODUCTION}

Chinese history architecture features its bright colors (Cruickshank, 2011) and colored patterns significantly contribute to that. As a special part of architectural heritage, the colored pattern is not only artwork to decorate the architecture, but information reflecting specific historical era, such as history and literary allusions and scene paintings. The Long Corridor of the Summer Palace is famous of the colorful oil colored patterns. There are over 14000 patterns in the Long Corridor throughout all the tie beams. However, due to the environment changes and ancient pigments and material, colored patterns are highly vulnerable to erosion and the conservation of colored patterns is not optimistic. So far, there seem to be no convenient and effect method to monitor the status quo of colored patterns. As a result, we proposed a method based on color analysis after verifying the precision and have implemented it in the Long Corridor of the Summer Place for four years. By detecting color information of sample points on colored patterns, color difference is calculated to evaluate the color decaying degree. This work can instruct the color restoration program as data source.

\section{EXPERIMENTAL PROCEDURE}

\subsection{Set up the illuminants}

When the sky turns totally dark, use standard illuminants D65 to light up the colored pattern. There should be even illuminance and no overexposure. Generally, two light tubes are placed symmetrically, and the extension of the tubes is at an angle of $45^{\circ}$ to the plane of the pattern. The height of the lights is approximately the same as the horizontal axis of the colored pattern and the distance between the lights and the colored pattern is $50-100 \mathrm{~cm}$. Based on the size of the pattern, the number of the light tubes is acceptable to change as long as the pattern can get soft and even illumination.

\subsection{Adjust the illuminants}

Slightly adjust the location and posture of the light sources and measure the illuminance distribution on the pattern to make sure the illuminance distribution is the same as the one of last detection. Since under the different illuminance, the detection lightness will be affected much, which will lead to great error of color analysis (Jiang, 1995). Thus, it is necessary to keep the illuminance constant.

The illuminance measuring procedure is as follows. A row of illuminance sensors are arranged at the bottom edge of the colored pattern surface where the distance between sensors is 5 $\mathrm{cm}$. Get the illuminance of the row by illuminometer. Then rise up the row of sensors for $10 \mathrm{~cm}$ and detect the illuminance until it goes to the top edge. As a result, the surface illuminance distribution matrix of the colored pattern is obtained. According to the illuminance matrix by the test and through the interpolation operation, the two-dimensional illuminance cloud image of the colored pattern surface and the numerical matrix A of the cloud image are generated on the computer. Matrix A is operated with the illuminance cloud image numerical matrix $\mathrm{A}_{0}$ for the first time monitoring:

Subtracting the corresponding values of the two matrices and calculating the standard deviation $\sigma$,

$$
\begin{gathered}
A=\left[\begin{array}{ccc}
\mathrm{y}_{11} & \cdots & \mathrm{y}_{\mathrm{i} 1} \\
\vdots & \ddots & \vdots \\
\mathrm{y}_{1 \mathrm{j}} & \cdots & \mathrm{y}_{\mathrm{ij}}
\end{array}\right], \mathrm{A}_{0}=\left[\begin{array}{ccc}
\mathrm{x}_{11} & \cdots & \mathrm{x}_{\mathrm{i} 1} \\
\vdots & \ddots & \vdots \\
\mathrm{x}_{1 \mathrm{j}} & \cdots & \mathrm{x}_{\mathrm{ij}}
\end{array}\right] \\
\sigma=\sqrt{\frac{1}{\mathrm{i} \cdot \mathrm{j}} \sum_{\substack{\mathrm{i}=1 \\
\mathrm{j}=1}}^{\mathrm{i} j}\left(\mathrm{y}_{\mathrm{ij}}-\mathrm{x}_{\mathrm{ij}}\right)^{2}}
\end{gathered}
$$




\subsection{Detect the color information}

According to $\sigma$ to adjust the location and posture of the symmetrical light sources. When the standard deviation $\sigma$ reaches the threshold, it can be regarded that the illuminance distributions of two tests are the same. The threshold is determined by the sensors' precision.

Third, detect the color of the colored pattern by 2D color analyzer and then get the tristimulus value and lightness in result. The analyzer is arranged on the central axis of the colored pattern.

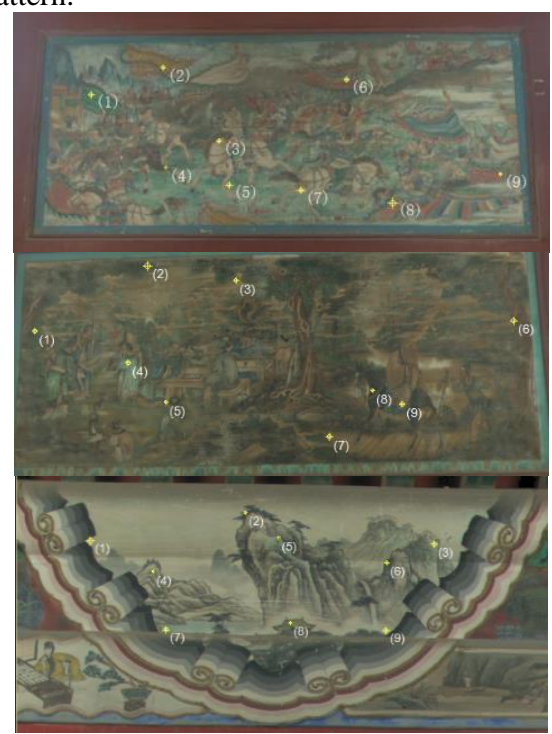

Figure 1. Examples of a colored pattern and sample points location distribution

\subsection{Calculate the color difference}

Calculate the color difference with the tristimulus value and values using CIE DE 2000 formula, by which the color decaying status of the patterns can be evaluated quantitatively. Nine sample points are selected from every colored pattern containing as many colors as possible and every point only contains a single color. Once the location of every sample point is determined, the tester should set sample points to the exact location in every subsequent test. There is a series of specific color information at every sample point, from which the tristimulus value $X, Y, Z$ and lightness $L$. are used for color difference calculation. The CIE DE2000 formula is as follows (CIE TC 1-47, 2001).

1. Calculate $L^{*}, a^{*}, b^{*}$ :

$$
\left.\begin{array}{c}
L^{*}=116 f\left(Y / Y_{n}\right)-16 \\
a^{*}=500\left[f\left(X / X_{n}\right)-f\left(Y / Y_{n}\right)\right] \\
b^{*}=200\left[f\left(Y / Y_{n}\right)-f\left(Z / Z_{n}\right)\right]
\end{array}\right\}
$$

where $f(t)=\left\{\begin{array}{cl}\sqrt[3]{t} & \text { if } t>\delta^{3} \\ \left(\frac{t}{3 \delta^{2}}\right)+\frac{4}{29} & \text { otherwise }\end{array}\right.$

$$
\delta=\frac{6}{29}
$$

$L^{*}, a^{*}, b^{*}=$ the coordination of a color in CIELAB color space

$X, Y, Z=$ the CIE XYZ tristimulus values of a color

$X_{n}, Y_{n}, Z_{n}=$ the CIE XYZ tristimulus values of the reference white point,

Under Illuminant D65 with normalization $\mathrm{Y}=100$, the values are $X_{n}=95.047, Y_{n}=100.000, Z_{n}=108.883$ where $C_{a b}^{*}=$ the CIELAB chroma

$$
C_{a b}^{*}=\sqrt{\left(a^{*}\right)^{2}+\left(b^{*}\right)^{2}}
$$

3. Calculate $L^{\prime}, a^{\prime}, b^{\prime}$ :

$$
a^{\prime}=a^{*}\left(\begin{array}{c}
L^{\prime}=L^{*} \\
\left.1+0.5\left(1-\sqrt{\frac{\bar{C}_{a b}^{* 7}}{\bar{C}_{a b}^{*}{ }^{7}+25^{7}}}\right)\right) \\
b^{\prime}=b^{*}
\end{array}\right.
$$

where $L^{\prime}, a^{\prime}, b^{\prime}=$ the modified coordination of a color in CIELAB color space

4. Calculate $C^{\prime}, h^{\prime}$ :

$$
\begin{aligned}
C^{\prime} & =\sqrt{\left(a^{\prime}\right)^{2}+\left(b^{\prime}\right)^{2}} \\
h^{\prime} & =\arctan \left(b^{\prime} / a^{\prime}\right)
\end{aligned}
$$

where $C^{\prime}, h^{\prime}=$ the modified CIELAB chroma and hue angle

5. Calculate $\Delta L^{\prime}, \Delta C^{\prime}, \Delta H^{\prime}$ :

$$
\begin{gathered}
\Delta L^{\prime}=L_{2}^{\prime}-L_{1}^{\prime} \\
\Delta C^{\prime}=C_{2}^{\prime}-C_{1}^{\prime} \\
\Delta H^{\prime}=2 \sqrt{C_{2}^{\prime}-C_{1}^{\prime}} \sin \left(\frac{h_{2}^{\prime}-h_{1}^{\prime}}{2}\right)
\end{gathered}
$$

where $\Delta L^{\prime}, \Delta C^{\prime}, \Delta H^{\prime}=$ the lightness, chroma and hue difference of two sample colors

6. Calculate the weighting functions, $S_{L}, S_{C}, S_{H}$ :

$$
\begin{gathered}
S_{L}=1+\frac{0.015\left(\bar{L}^{\prime}-50\right)^{2}}{\sqrt{20+\left(\bar{L}^{\prime}-50\right)^{2}}} \\
S_{C}=1+0.045 \bar{C}^{\prime} \\
S_{H}=1+0.015 \bar{C}^{\prime} T \\
T=1-0.17 \cos \left(\bar{h}^{\prime}-30\right)+0.24 \cos \left(2 \bar{h}^{\prime}\right) \\
+0.32 \cos \left(3 \bar{h}^{\prime}+6\right)-0.20 \cos \left(4 \bar{h}^{\prime}+63\right)
\end{gathered}
$$

where $S_{L}, S_{C}, S_{H}=$ the weighting functions of the lightness, chroma and hue

$\bar{L}^{\prime}, \bar{C}^{\prime}, \bar{h}^{\prime}=$ the arithmetic mean of the corresponding values of the colour-difference pair.

7. Calculate the rotation function, $R_{T}$ :

$$
\begin{gathered}
R_{T}=-\sin (2 \Delta \Theta) R_{C} \\
\Delta \Theta=30 \exp \left\{-\left[\left(\bar{h}^{\prime}-275\right) / 25\right]^{2}\right\} \\
R_{C}=2 \sqrt{\frac{\bar{C}^{\prime 7}}{\bar{C}^{\prime 7}+25^{7}}}
\end{gathered}
$$

where $R_{T}=$ the rotation function

$$
\bar{h}^{\prime} \text { and } \Delta \Theta \text { are in degree units. }
$$

\section{Calculate CIE DE2000 Color Difference $\Delta E_{00}$ :}

The total colour-difference between two colour samples with lightness, chroma and hue differences, $\Delta L^{\prime}, \Delta C^{\prime}, \Delta H^{\prime}$, with weighting functions, $S_{L}, S_{C}, S_{H}$, parametric factors, $k_{L}, k_{C}, k_{H}$ and rotation function $R_{T}$ is determined by Equation .

$$
=\sqrt{\left(\frac{\Delta L^{\prime}}{S_{L} k_{L}}\right)^{2}+\left(\frac{\Delta C^{\prime}}{S_{C} k_{C}}\right)^{2}+\left(\frac{\Delta H^{\prime}}{S_{H} k_{H}}\right)^{2}+R_{T}\left(\frac{\Delta C^{\prime}}{S_{C} k_{C}}\right)\left(\frac{\Delta H^{\prime}}{S_{H} k_{H}}\right)}
$$

where parametric factors $k_{L}, k_{C}, k_{H}=1$

\section{Calculate $C_{a b}^{*}$ :}


The monitoring cycle should be at least 3 months. The color evaluate the color decaying status of the pattern. difference at every spot between two tests are calculated to

\begin{tabular}{|c|c|c|c|c|c|c|c|c|c|c|c|c|}
\hline \multirow[b]{2}{*}{ Tester } & \multicolumn{4}{|c|}{$\mathrm{X}$} & \multicolumn{4}{|c|}{$\mathrm{Y}$} & \multicolumn{4}{|c|}{ Z } \\
\hline & $1 \mathrm{st}$ & 2 nd & error & $\begin{array}{l}\text { relative } \\
\text { error }\end{array}$ & $1 \mathrm{st}$ & 2 nd & error & $\begin{array}{l}\text { relative } \\
\text { error }\end{array}$ & $1 \mathrm{st}$ & 2nd & error & $\begin{array}{c}\text { relative } \\
\text { error }\end{array}$ \\
\hline \multirow{5}{*}{1} & 12.533 & 12.533 & 0 & $0 \%$ & 14.66 & 14.66 & 0 & $0 \%$ & 13.2345 & 13.2345 & 0 & $0 \%$ \\
\hline & 5.5279 & 5.4112 & 0.1167 & $2 \%$ & 6.5978 & 6.4512 & 0.1466 & $2 \%$ & 8.127 & 8.0519 & 0.0751 & $1 \%$ \\
\hline & 10.6599 & 10.5001 & 0.1598 & $1 \%$ & 11.8123 & 11.6356 & 0.1767 & $1 \%$ & 10.1075 & 9.9759 & 0.1316 & $1 \%$ \\
\hline & 4.0514 & 3.9722 & 0.0792 & $2 \%$ & 4.5509 & 4.4764 & 0.0745 & $2 \%$ & 4.6696 & 4.5968 & 0.0728 & $2 \%$ \\
\hline & 6.3628 & 6.3628 & 0 & $0 \%$ & 6.2593 & 6.2593 & 0 & $0 \%$ & 4.6074 & 4.6074 & 0 & $0 \%$ \\
\hline \multirow{5}{*}{2} & 12.5685 & 12.6074 & 0.0389 & $0 \%$ & 14.6418 & 14.7583 & 0.1165 & $1 \%$ & 13.2727 & 13.2993 & 0.0266 & $0 \%$ \\
\hline & 5.711 & 5.4112 & 0.2998 & $5 \%$ & 6.8306 & 6.4512 & 0.3794 & $6 \%$ & 8.2869 & 8.0519 & 0.235 & $3 \%$ \\
\hline & 10.7161 & 10.5645 & 0.1516 & $1 \%$ & 11.9046 & 11.7373 & 0.1673 & $1 \%$ & 10.1752 & 10.0713 & 0.1039 & $1 \%$ \\
\hline & 4.1308 & 4.0351 & 0.0957 & $2 \%$ & 4.6524 & 4.5547 & 0.0977 & $2 \%$ & 4.7366 & 4.6412 & 0.0954 & $2 \%$ \\
\hline & 6.7419 & 6.5389 & 0.203 & $3 \%$ & 6.5796 & 6.3893 & 0.1903 & $3 \%$ & 4.7224 & 4.6638 & 0.0586 & $1 \%$ \\
\hline \multirow{5}{*}{3} & 12.533 & 12.6947 & 0.1617 & $1 \%$ & 14.66 & 14.8908 & 0.2308 & $2 \%$ & 13.2345 & 13.3446 & 0.1101 & $1 \%$ \\
\hline & 5.666 & 5.5631 & 0.1029 & $2 \%$ & 6.7626 & 6.6405 & 0.1221 & $2 \%$ & 8.2136 & 8.2039 & 0.0097 & $0 \%$ \\
\hline & 10.4159 & 10.5645 & 0.1486 & $1 \%$ & 11.5847 & 11.7373 & 0.1526 & $1 \%$ & 9.9498 & 10.0713 & 0.1215 & $1 \%$ \\
\hline & 4.4776 & 3.9722 & 0.5054 & $11 \%$ & 5.055 & 4.4764 & 0.5786 & $11 \%$ & 5.1268 & 4.5968 & 0.53 & $10 \%$ \\
\hline & 6.1985 & 6.4763 & 0.2778 & $4 \%$ & 6.1081 & 6.285 & 0.1769 & $3 \%$ & 4.5581 & 4.6033 & 0.0452 & $1 \%$ \\
\hline \multirow{5}{*}{4} & 12.6561 & 12.6074 & 0.0487 & $0 \%$ & 14.8196 & 14.7583 & 0.0613 & $0 \%$ & 13.3581 & 13.2993 & 0.0588 & $0 \%$ \\
\hline & 5.666 & 5.666 & 0 & $0 \%$ & 6.7626 & 6.7626 & 0 & $0 \%$ & 8.2136 & 8.2136 & 0 & $0 \%$ \\
\hline & 10.0204 & 9.8794 & 0.141 & $1 \%$ & 11.0728 & 10.9188 & 0.154 & $1 \%$ & 9.5089 & 9.3868 & 0.1221 & $1 \%$ \\
\hline & 4.0301 & 4.0303 & 0.0002 & $0 \%$ & 4.5054 & 4.5389 & 0.0335 & $1 \%$ & 4.6481 & 4.6433 & 0.0048 & $0 \%$ \\
\hline & 6.3373 & 6.3199 & 0.0174 & $0 \%$ & 6.2122 & 6.1778 & 0.0344 & $1 \%$ & 4.6 & 4.5969 & 0.0031 & $0 \%$ \\
\hline
\end{tabular}

Table 1. Sample points placing error analysis of the east side pattern in Liujia Pavilion

\begin{tabular}{|c|c|c|c|c|c|c|c|c|c|c|c|c|}
\hline \multirow[b]{2}{*}{ Tester } & \multicolumn{4}{|c|}{$\mathrm{X}$} & \multicolumn{4}{|c|}{$\mathrm{Y}$} & \multicolumn{4}{|c|}{ Z } \\
\hline & $1 \mathrm{st}$ & 2nd & error & $\begin{array}{c}\text { relative } \\
\text { error }\end{array}$ & $1 \mathrm{st}$ & $2 \mathrm{nd}$ & error & $\begin{array}{c}\text { relative } \\
\text { error }\end{array}$ & $1 \mathrm{st}$ & 2nd & error & $\begin{array}{c}\text { relative } \\
\text { error }\end{array}$ \\
\hline \multirow{5}{*}{1} & 8.7917 & 8.7917 & 0 & $0 \%$ & 9.308 & 9.308 & 0 & $0 \%$ & 6.8463 & 6.8463 & 0 & $0 \%$ \\
\hline & 7.3013 & 7.0648 & 0.2365 & $3 \%$ & 7.8496 & 7.6418 & 0.2078 & $3 \%$ & 5.5937 & 5.3918 & 0.2019 & $4 \%$ \\
\hline & 6.5511 & 6.542 & 0.0091 & $0 \%$ & 7.1081 & 7.1018 & 0.0063 & $0 \%$ & 4.9734 & 4.9505 & 0.0229 & $0 \%$ \\
\hline & 5.6972 & 5.6539 & 0.0433 & $1 \%$ & 6.1113 & 6.0803 & 0.031 & $1 \%$ & 4.2929 & 4.2593 & 0.0336 & $1 \%$ \\
\hline & 2.9666 & 2.9666 & 0 & $0 \%$ & 3.2836 & 3.2836 & 0 & $0 \%$ & 2.5379 & 2.5379 & 0 & $0 \%$ \\
\hline \multirow{5}{*}{2} & 9.3458 & 8.6692 & 0.6766 & $7 \%$ & 9.9512 & 9.1184 & 0.8328 & $8 \%$ & 7.4384 & 6.6868 & 0.7516 & $10 \%$ \\
\hline & 7.6493 & 6.8296 & 0.8197 & $11 \%$ & 8.2408 & 7.3908 & 0.85 & $10 \%$ & 5.8469 & 5.1947 & 0.6522 & $11 \%$ \\
\hline & 6.6776 & 6.4884 & 0.1892 & $3 \%$ & 7.3072 & 7.0504 & 0.2568 & $4 \%$ & 5.0757 & 4.8963 & 0.1794 & $4 \%$ \\
\hline & 4.9173 & 5.4491 & 0.5318 & $11 \%$ & 5.2379 & 5.8445 & 0.6066 & $12 \%$ & 3.5574 & 4.0828 & 0.5254 & $15 \%$ \\
\hline & 3.1851 & 2.9184 & 0.2667 & $8 \%$ & 3.505 & 3.2425 & 0.2625 & $7 \%$ & 2.7804 & 2.4968 & 0.2836 & $10 \%$ \\
\hline \multirow{5}{*}{3} & 8.6818 & 8.9645 & 0.2827 & $3 \%$ & 9.1543 & 9.5144 & 0.3601 & $4 \%$ & 6.7346 & 7.0163 & 0.2817 & $4 \%$ \\
\hline & 7.1872 & 7.1238 & 0.0634 & $1 \%$ & 7.7568 & 7.7133 & 0.0435 & $1 \%$ & 5.4982 & 5.4292 & 0.069 & $1 \%$ \\
\hline & 6.5039 & 6.542 & 0.0381 & $1 \%$ & 7.0455 & 7.1018 & 0.0563 & $1 \%$ & 4.9209 & 4.9505 & 0.0296 & $1 \%$ \\
\hline & 5.4226 & 5.4877 & 0.0651 & $1 \%$ & 5.8146 & 5.876 & 0.0614 & $1 \%$ & 4.0618 & 4.1097 & 0.0479 & $1 \%$ \\
\hline & 2.9685 & 2.96 & 0.0085 & $0 \%$ & 3.2831 & 3.3045 & 0.0214 & $1 \%$ & 2.5159 & 2.4952 & 0.0207 & $1 \%$ \\
\hline \multirow{5}{*}{4} & 8.6818 & 8.7989 & 0.1171 & $1 \%$ & 9.1543 & 9.286 & 0.1317 & $1 \%$ & 6.7346 & 6.8225 & 0.0879 & $1 \%$ \\
\hline & 7.1238 & 7.2017 & 0.0779 & $1 \%$ & 7.7133 & 7.7617 & 0.0484 & $1 \%$ & 5.4292 & 5.5083 & 0.0791 & $1 \%$ \\
\hline & 6.4412 & 6.542 & 0.1008 & $2 \%$ & 6.97 & 7.1018 & 0.1318 & $2 \%$ & 4.897 & 4.9505 & 0.0535 & $1 \%$ \\
\hline & 5.5641 & 5.4393 & 0.1248 & $2 \%$ & 5.972 & 5.8314 & 0.1406 & $2 \%$ & 4.1923 & 4.0912 & 0.1011 & $2 \%$ \\
\hline & 2.9429 & 2.9631 & 0.0202 & $1 \%$ & 3.2597 & 3.2702 & 0.0105 & $0 \%$ & 2.5063 & 2.562 & 0.0557 & $2 \%$ \\
\hline
\end{tabular}

Table 2. Sample points placing error analysis of the west side pattern in Liujia Pavilion 


\section{SAMPLE POINTS PLACING ERROR ANALYSIS}

Since placing sample points is conducted by the tester, some accidental error does exist in every time. To verify the placing chose two colored patterns in Liujia Pavilion, and each pattern tested twice. Every volunteer should place the sample points to fixed locations twice for one colored pattern. Thus, we can analyze the repetitively placing error. The results are shown in Table 1 and Table 2. error, four volunteers are invited to operate the procedure. We

As we can see from the tables, despite Tester 2, the sample points placing error is from 0 to $3 \%$, which is small enough and will not influence the accuracy of color difference calculation.

\section{COLOR DIFFERENCE RESULTS}

The color differences and the averages of nine sample points on one pattern are calculated for every quarter and every year. Due to the space limitation, only the averages (without the max and min values) and medians of 4 patterns (totally 16) are listed in Table 3 and Figure 2. The pictures of the four patterns are shown in Figure 3.

\begin{tabular}{|c|c|c|c|c|c|c|c|c|c|c|c|}
\hline $\begin{array}{c}\text { Year- } \\
\text { Quarter }\end{array}$ & 1 & 2 & 3 & 4 & 5 & 6 & 7 & 8 & 9 & Median & Average \\
\hline \multirow{4}{*}{$2016-2$} & 2.074 & 1.422 & 2.471 & 1.471 & 1.858 & 2.681 & 3.467 & 4.176 & 4.677 & 2.471 & 2.600 \\
\hline & 4.557 & 5.676 & 3.232 & 5.053 & 4.681 & 3.534 & 2.913 & 3.036 & 2.203 & 3.534 & 3.858 \\
\hline & 1.946 & 1.273 & 1.332 & 2.142 & 1.945 & 1.813 & 4.105 & 2.383 & 1.067 & 1.945 & 1.833 \\
\hline & 1.657 & 3.532 & 5.074 & 1.641 & 2.553 & 3.791 & 2.860 & 4.022 & 4.787 & 3.532 & 3.315 \\
\hline \multirow{4}{*}{$2016-3$} & 6.453 & 3.339 & 4.835 & 5.858 & 3.540 & 3.613 & 3.496 & 3.220 & 6.524 & 3.613 & 4.448 \\
\hline & 3.184 & 3.457 & 2.657 & 5.920 & 3.336 & 4.102 & 3.301 & 4.318 & 4.710 & 3.457 & 3.773 \\
\hline & 2.734 & 1.208 & 2.938 & 2.448 & 2.475 & 2.854 & 3.034 & 2.388 & 2.376 & 2.475 & 2.602 \\
\hline & 3.526 & 2.530 & 1.991 & 4.647 & 1.781 & 3.188 & 0.540 & 2.059 & 4.934 & 2.530 & 2.817 \\
\hline \multirow{4}{*}{$2016-4$} & 2.856 & 1.037 & 4.568 & 3.797 & 2.301 & 3.379 & 6.813 & 4.617 & 6.048 & 3.797 & 3.938 \\
\hline & 1.318 & 1.356 & 5.176 & 4.133 & 1.916 & 5.886 & 5.672 & 4.405 & 5.908 & 4.405 & 4.078 \\
\hline & 1.978 & 5.371 & 4.397 & 4.530 & 3.227 & 2.917 & 5.728 & 4.112 & 6.822 & 4.397 & 4.326 \\
\hline & 1.920 & 4.119 & 8.745 & 5.076 & 2.352 & 3.183 & 5.117 & 5.149 & 3.764 & 4.119 & 4.109 \\
\hline \multirow{4}{*}{2016} & 10.700 & 3.284 & 12.664 & 12.452 & 2.794 & 2.816 & 1.918 & 3.810 & 5.584 & 3.810 & 5.920 \\
\hline & 4.993 & 2.475 & 15.390 & 6.291 & 2.197 & 2.056 & 2.332 & 4.538 & 5.812 & 4.538 & 4.091 \\
\hline & 6.754 & 7.339 & 8.796 & 6.410 & 4.404 & 2.022 & 2.861 & 3.350 & 6.380 & 6.380 & 5.357 \\
\hline & 9.544 & 3.810 & 15.552 & 5.000 & 2.210 & 1.968 & 2.164 & 3.832 & 3.502 & 3.810 & 4.295 \\
\hline \multirow{4}{*}{$2017-1$} & 4.117 & 2.444 & 1.933 & 1.445 & 0.767 & 2.977 & 2.125 & 0.942 & 2.055 & 2.055 & 1.989 \\
\hline & 4.014 & 1.414 & 1.181 & 1.205 & 0.488 & 3.422 & 1.872 & 1.146 & 1.872 & 1.414 & 1.730 \\
\hline & 2.722 & 2.682 & 1.490 & 0.319 & 0.861 & 2.258 & 2.709 & 1.521 & 1.801 & 1.801 & 1.903 \\
\hline & 3.557 & 2.048 & 2.993 & 0.565 & 0.667 & 1.752 & 1.297 & 1.628 & 1.881 & 1.752 & 1.752 \\
\hline \multirow{4}{*}{$2017-2$} & 12.557 & 10.401 & 9.208 & 10.473 & 8.199 & 11.644 & 8.569 & 8.044 & 7.653 & 9.208 & 9.505 \\
\hline & 10.472 & 6.603 & 6.611 & 10.589 & 7.518 & 5.832 & 6.719 & 8.183 & 6.646 & 6.719 & 7.536 \\
\hline & 10.354 & 2.299 & 6.486 & 9.454 & 10.167 & 3.639 & 8.397 & 5.972 & 5.283 & 6.486 & 7.057 \\
\hline & 13.802 & 4.302 & 12.867 & 13.940 & 7.522 & 5.553 & 9.208 & 7.426 & 5.660 & 7.522 & 8.863 \\
\hline \multirow{4}{*}{$2017-3$} & 1.246 & 1.552 & 10.059 & 9.882 & 8.211 & 3.192 & 3.366 & 1.153 & 10.337 & 3.366 & 5.358 \\
\hline & 1.711 & 0.829 & 10.007 & 6.732 & 2.278 & 2.099 & 0.691 & 3.096 & 6.270 & 2.278 & 3.288 \\
\hline & 1.444 & 3.289 & 8.556 & 3.312 & 2.715 & 5.313 & 3.084 & 0.665 & 7.255 & 3.289 & 3.773 \\
\hline & 3.329 & 4.357 & 11.005 & 2.682 & 4.991 & 3.244 & 2.040 & 0.764 & 1.947 & 3.244 & 3.227 \\
\hline \multirow{4}{*}{$2017-4$} & 0.730 & 15.684 & 9.521 & 5.798 & 7.198 & 2.343 & 7.523 & 4.716 & 2.134 & 5.798 & 5.605 \\
\hline & 1.054 & 9.623 & 7.790 & 8.015 & 4.434 & 3.811 & 5.377 & 6.840 & 1.510 & 5.377 & 5.397 \\
\hline & 5.724 & 11.655 & 8.230 & 6.094 & 2.098 & 1.837 & 8.817 & 5.728 & 0.600 & 5.728 & 5.504 \\
\hline & 0.331 & 14.679 & 5.728 & 7.342 & 3.002 & 2.574 & 6.407 & 8.095 & 1.359 & 5.728 & 4.930 \\
\hline \multirow{4}{*}{2017} & 2.607 & 11.396 & 9.013 & 9.011 & 7.272 & 2.087 & 8.939 & 7.661 & 8.357 & 8.357 & 7.551 \\
\hline & 0.733 & 8.221 & 5.626 & 12.326 & 5.763 & 1.980 & 7.250 & 3.118 & 7.492 & 5.763 & 5.636 \\
\hline & 1.081 & 7.788 & 7.740 & 8.132 & 7.587 & 2.007 & 6.660 & 0.524 & 7.498 & 7.498 & 5.766 \\
\hline & 3.851 & 17.192 & 4.999 & 8.152 & 3.432 & 1.908 & 9.911 & 2.651 & 7.199 & 4.999 & 5.742 \\
\hline \multirow{4}{*}{ 2018-1 } & 1.131 & 11.878 & 13.264 & 8.074 & 4.494 & 4.703 & 3.677 & 2.218 & 6.584 & 4.703 & 5.947 \\
\hline & 2.236 & 8.381 & 13.101 & 6.917 & 6.301 & 3.267 & 6.849 & 2.393 & 7.719 & 6.849 & 5.975 \\
\hline & 3.257 & 10.550 & 10.750 & 8.950 & 4.258 & 5.244 & 4.367 & 3.974 & 7.256 & 5.244 & 6.371 \\
\hline & 2.440 & 6.731 & 12.232 & 4.862 & 5.186 & 6.120 & 5.281 & 3.104 & 10.054 & 5.281 & 5.905 \\
\hline
\end{tabular}


The International Archives of the Photogrammetry, Remote Sensing and Spatial Information Sciences, Volume XLII-2/W11, 2019 GEORES 2019 - 2nd International Conference of Geomatics and Restoration, 8-10 May 2019, Milan, Italy

\begin{tabular}{llllllllllll}
\hline \multirow{2}{*}{$2018-2$} & 0.765 & 9.819 & 7.752 & 7.868 & 4.784 & 1.933 & 1.622 & 6.193 & 9.994 & 6.193 & 5.710 \\
& 1.061 & 11.122 & 8.264 & 5.000 & 3.185 & 0.844 & 6.910 & 4.320 & 0.668 & 4.320 & 4.226 \\
& 1.998 & 12.162 & 7.605 & 4.833 & 2.018 & 0.321 & 2.219 & 2.492 & 5.612 & 2.492 & 3.825 \\
& 1.331 & 12.908 & 13.543 & 2.400 & 4.352 & 1.624 & 1.569 & 0.415 & 9.274 & 2.400 & 4.780 \\
\hline \multirow{2}{*}{$2018-3$} & 1.281 & 10.840 & 10.722 & 4.488 & 2.441 & 9.908 & 2.670 & 2.470 & 12.700 & 4.488 & 6.220 \\
& 2.927 & 10.453 & 12.158 & 5.136 & 2.212 & 2.013 & 1.568 & 3.787 & 8.658 & 3.787 & 5.027 \\
& 2.070 & 11.257 & 11.855 & 3.972 & 8.557 & 6.368 & 1.093 & 2.911 & 3.827 & 3.972 & 5.566 \\
$2018-4$ & 1.240 & 10.226 & 15.362 & 6.816 & 6.232 & 7.897 & 1.423 & 2.823 & 3.810 & 6.232 & 5.604 \\
\hline & 3.498 & 12.544 & 6.263 & 9.051 & 1.505 & 8.108 & 6.654 & 0.723 & 2.102 & 6.263 & 5.312 \\
& 3.153 & 10.098 & 6.462 & 11.078 & 1.629 & 6.103 & 10.877 & 3.571 & 3.244 & 6.103 & 6.215 \\
& 1.151 & 7.809 & 3.705 & 9.917 & 1.613 & 6.816 & 6.003 & 1.470 & 2.262 & 3.705 & 4.337 \\
& 1.917 & 9.491 & 5.350 & 12.716 & 2.104 & 4.134 & 6.863 & 0.552 & 2.544 & 4.134 & 4.629 \\
\hline \multirow{6}{*}{2018} & 0.732 & 14.573 & 7.818 & 3.913 & 2.444 & 1.758 & 2.401 & 12.340 & 0.454 & 2.444 & 4.487 \\
& 0.525 & 12.013 & 2.943 & 4.394 & 1.089 & 2.134 & 0.656 & 13.218 & 3.457 & 2.943 & 3.812 \\
& 0.720 & 10.897 & 5.081 & 2.935 & 3.978 & 1.827 & 9.054 & 11.467 & 2.239 & 3.978 & 5.144 \\
& 1.577 & 10.602 & 7.806 & 3.847 & 1.211 & 1.853 & 4.033 & 10.742 & 0.948 & 3.847 & 4.418 \\
\hline
\end{tabular}

Table 3. The averages and medians of $\Delta E_{00}$ in four colored patterns

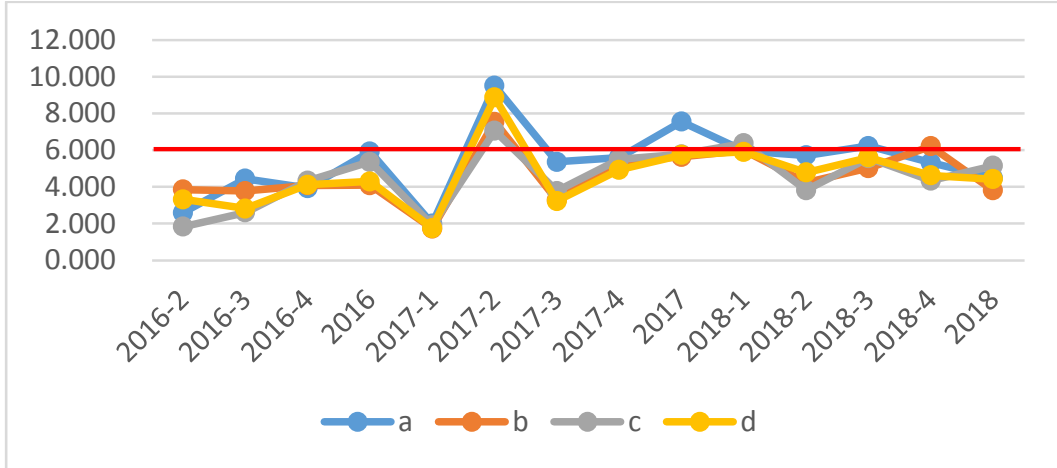

Figure 2. The averages and medians of $\Delta E_{00}$ in four colored patterns

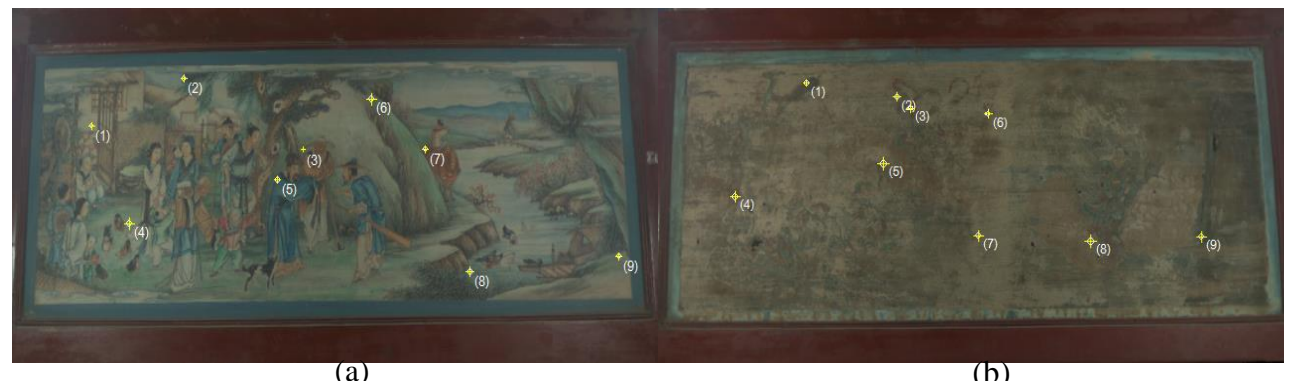

(a)

(b)

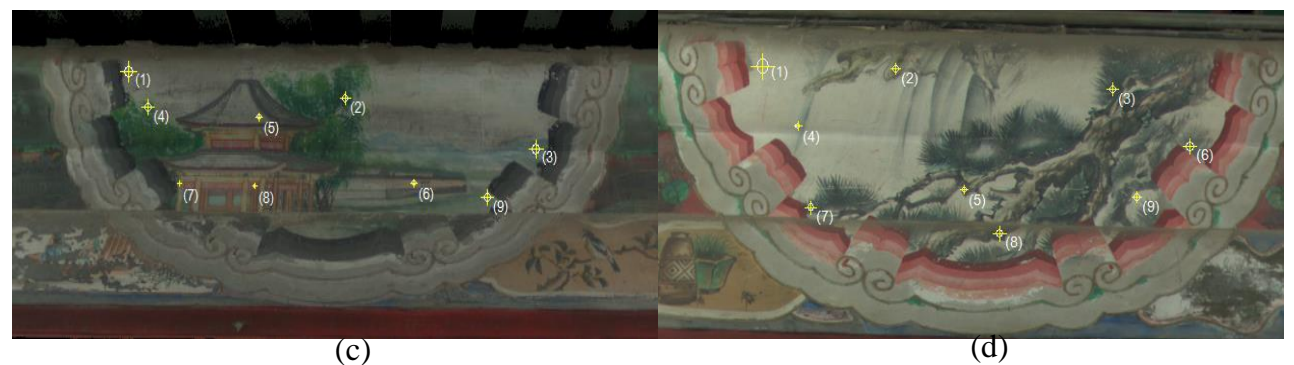

Figure 3. Pictures of the four colored pattern

(a) East side of Jilan Pavilion, (b) West side of Jilan Pavilion,

(c) Outside (facing south) of a corridor tie beam, (d) Inside (facing north) of the corridor tie beam

The color differences $\Delta E_{00}$ are mainly in the range from 2 to 6 . Some even can be over 9 . Although the values are kind of large for other industry, there seem to be no large changes from the patterns. For most colored patterns, they still stay in good 
condition and look colorful. So the corresponding standard need to be formulated. Based on the work experience these years, we have a proposing standard. When the average color difference is between 0 and 6 , there are some slight changes that people can hardly notice. When it is between 6 and 12, there are some changes that people can be aware of. When it is over 15, there are obvious changes that people can find easily. And further research need to be done to verify the standard. As a result, color decaying happens every time, and there are slight color changes on most colored patterns. In $2^{\text {nd }}$ quarter 2017, aware color changes happened on nearly all the patterns.

On the other hand, it is obviously that the conservation status of east side in Liujia Pavilion is much better than the one of west side from the picture. The reason is that the sunlight shines on the Kunming Lake in the morning and the water (ice in winter) reflects the light on the west side pattern. When the sun sets, the light is too weak to affect the pattern and plants beside the lake can exactly block the light (Zhang, 2017). From the data, the fact is the $\Delta E_{00}$ of east side pattern is usually larger than the $\triangle E_{00}$ of west side pattern. It is because the west side one has been badly decaying and is hard to change much. So this work has better begin from finishing painting and last for all life cycle of them. Then more instructive data can be gotten.

\section{CONCLUSION}

To monitor the color decaying condition of the colored patterns, a convenient and effective method is drawn up. By detecting the color information, color differences can be calculate with CIE DE2000 formula. The color decaying condition can be evaluated and the data can instruct the maintenance work and the evaluating standard still need more research to verify. From our monitor, most colored patterns keep in good condition and only slight color changes happened. The monitor work has better begin when the colored patterns are finished drawing and last for the all life cycle. So more instructive data can be gotten.

\section{ACKNOWLEDGEMENTS}

This work is partially supported by the Management Office of the Summer Palace.

\section{REFERENCE}

CIE Technical Committee 1-47 of Division 1 "Vision and Colour", 2001. Improvement to Industrial Colour-Difference Evaluation. COMMISSION INTERNATIONALE DE L'ECLAIRAGE, CIE Central Bureau, Vienna, Austria, 2-6.

Cruickshank, D., 2011. Sir Banister Fletcher's A History of Architecture. 20th ed. Intellectual Property Publishing House, Beijing, China, 724.

J., Jiang, et al, 1995. Applied Colorimetry. Chinese People's Liberation Army Publishing House, Beijing, China, 115-117.

L. Zhang, Z. Wang, X. Zhao, et al, 2017. The Monitoring and Analysis of the Chinese Traditional Architecture Painting Decay - the Case of Jilan Pavilion in the Summer Palace. The International Archives of the Photogrammetry, Remote Sensing and Spatial Information Sciences, Vol XLII-2-W5, 737-741. https://doi.org/10.5194/isprs-archives-XLII-2-W5-737-2017. 